\title{
Impact of Educational Program about Food Safety on Improving Adolescent Students' Knowledge and Practices in Assiut
}

\author{
Amina Mohamed Thabet \& Nora Abd-Elhamid Zaki \\ Lecturer of pediatric nursing- Sohag University \\ Lecturer of pediatric nursing- Assuit University
}

\begin{abstract}
Food safety is a global health goal and food borne diseases are a major health issue. It is important to reach young adults with food safety education because of their current and future roles as caregivers. The aim of this study was to establish baseline assessment data regarding to food safety knowledge and practices among adolescent students and develop an educational program on the base of previous baseline data to increase their knowledge and improve their practices related to food safety. A quasi-experimental study design was used in carrying out at 4 secondary schools (two schools were east and the others were west) in Assuit. The sample included 1000 students (as baseline assessment data) and 200 students randomly selected for the educational program. The main result in this study was no statistically significant differences between the pre test and post test practices and knowledge in the control group but there was statistically significant difference between the pre-post test practices and knowledge in the study group. The study was concluded that, the students' knowledge and practices about food safety were increased significantly among students who had the food safety educational program. It is recommended that the developed programs should be taught among different age group students that do not offer food safety program.
\end{abstract}

\section{Keywords: Food safety, Knowledge, practices, Food borne diseases, Secondary schools \& adolescent students}

\section{Introduction}

Food safety is a scientific discipline describing handling, preparation, and storage of food in ways that prevent food borne illness. According to the World Health Organization (WHO) in the year 2010 number of routines that should be followed to avoid potentially severe health hazards. The five key principles of food hygiene, Prevent contaminating food with pathogens spreading from people, pets, and pests, separate raw and cooked foods to prevent contaminating the cooked foods, cook foods for the appropriate length of time and at the appropriate temperature to kill pathogens, store food at the proper temperature, and the use of safe water and cooked materials food borne diseases represent a widespread and growing public health problem, in both developed and developing countries. This problem, however, has more impact on health and economy in developing countries but no reliable data are available (WHO, 2007).

Egypt is becoming aware of the importance of food safety to its people, and for its well-being as a nation. However, it is important to note that Egypt is not alone in these concerns. The global problem of food safety requires a global approach. Egypt move forward as a nation to help solve this worldwide problem. Egypt is joining the growing number of nations who are creating, rational, science-based food safety strategies to protect public health. This is the right thing to do for public health. Ensuring food safety is critical for all of us and a healthy populace will enhance all other public health and safety initiatives. The worldwide food safety problem will be solved as each country advances its own reforms. Kids are disproportionately affected by serious food borne illness. Of the 48 million reported food borne illnesses confirmed annually in the United States, half are among children under 15 years of age (The Partnership for Food Safety Education, 2013).

Food safety is the daily responsibility of those who prepare and serve food. Disease causing microorganisms on the body can come in contact with food or food contact surfaces and contaminate the food. Activities such as smoking, eating, drinking, handling raw foods, dispensing garbage, using the restroom, and touching the face are all sources of possible contamination. This contamination can make others sick. By practicing good personal hygiene and following food safety practices, the risk of a food borne illness can be reduced (Diane_et al., 2013). Teaching food safety is important, the good news is that there are many things children and families can do to help ensure that their food is safe to eat at home and at school and even when eating out. All they need is a basic awareness of proper food handling, cleanliness practices, and the importance of temperature in controlling/killing bacteria (Ajello et al., 2014). 
The need for enhancing food safety education has been known in developed countries with the launch of national initiatives to find ways to effectively educate consumers, especially the young, who prepare food. The changing demography and lifestyle, as well as emergence of resistant and exceptionally hazardous strains of food borne microorganisms, create a situation that could lead to major outbreaks of life threatening food borne illness (Haapala \& Probart, 2004). There is no education program for improving the food safety knowledge and practices of consumers in any age group or in schools, and assessing the basic knowledge of young consumers is essential for developing effective food safety programs. Food safety education is a fundamental aspect of the overall food safety initiative. Without knowledge of food safety practices and proper food handling procedures, food borne illnesses cannot be reduced (Redmond \& Griffith, 2005).

Effective educational interventions for adolescents can lead to improved food safety habits, but the success of these interventions depends upon alignment of educational strategies with specific needs of the targeted demographic group. Research suggests that without baseline data, it is difficult to develop and implement effective educational efforts. Constructing a baseline of food safety knowledge, attitudes, and behaviors for various demographic groups is vital for determining the specific educational strategies that will motivate adolescents to practice safer food-handling (Spedigo et al., 2009). Because data concerning adolescents' students' knowledge and practices are few, this study was conducted to evaluate the impact of an educational program on improving adolescents' knowledge and practices.

\section{Aim of the study}

The aim of this study was to:

Investigate the impact of educational program about food safety on improving adolescent students' knowledge and practices in Assuit.

\section{Hypothesis}

Adolescents' who attend the educational program about food safety exhibit better knowledge and practices than who do not.

\section{Subjects and Method}

Design: A quasi-experimental study design was used in carrying out this study

Setting: The study was conducted in 4 secondary schools (two schools were in west and the others were in east) in Assiut, El-Weledia PreparatorySecondary School for girls and E-Nasir Secondary School for boys (from the west) and The Secondary
University School for boys and Khadega Youssef Secondary School for girls (from the east).

Subject: The target population of this research consisted of secondary schools adolescence students. The sample was selected randomly to conduct the study. Their number amounted to1000 students (250 students from each school).

Tools of the study: Two tools were designed to collect the necessary data about students' safety knowledge and practices as follow:

Tool I: Knowledge and practice regarding food safety structured interview schedule, the tool was developed by the researchers after review of the literature to collect data about students' knowledge and practice regarding food safety. It included three parts as follows:

Part 1: Socio-demographic data about the students and their families as age, gender, mothers' and fathers' education, and mothers' occupation.

Part 2: It consisted of twenty six questions about knowledge regarding food safety. Questions are divided into five sections namely: general food safety knowledge, personal hygiene knowledge, cooking foods adequately knowledge, cross contamination knowledge, and knowledge about keeping the food at safe temperature.

Part 3: It contained thirty one questions about self reporting practices regarding food safety. Questions are divided into four sections namely: personal hygiene practices, cooking foods adequately practices, cross contamination practices, and practices about keeping the food at safe temperature.

Tool II: Socio-economic status of the family assessment scale was assessed using Abdel-Twab Scale 2002. It included questions about: The educational, occupational, financial status of the family, as well as the family life style.

\section{Method of data collection:}

-An official permission was granted from responsible personnel to carry out the study after explaining the purpose of study.

- Meetings with school managers to explain the objectives and contents of the program and the methods for applying the program were helpful for to gain their cooperation and to allow the release of students to attend the program.

- Complete confidentiality of any obtained information was ensured.

- A pilot study was carried out on a sample of 100 students. These students were excluded from the study. The aim of the pilot was to test the validity and reliability of the tools. It also helped to determine the time needed for filling up the questionnaire and to make the needed correction.

-The time needed to fill out such questionnaire in one session was 25-30 minutes. Following the pilot 
study, the questionnaire was finalized and made ready for use.

- Validity of tool one was estimated by 5 experts in pediatric field and its result was $92 \%$.

- Reliability was estimated by Alpha Cronbach's test for tool one and its result was $\mathrm{R}=0.84$.

Base line assessment of knowledge and practice was obtained using tool one.

- The questionnaire was self-administered to the 1000 students by the researchers who were interviewed individually in their schools during break times).

- Items in the questionnaire were explained by the researchers when necessary and administered at one sitting as far as possible.

- After assessment of the baseline data on fourty percent of the students, we randomly selected 50 students from each school to attend the educational program, after that the students were divided randomly into two groups (the first group was categorized for the control group and the second was categorized for the study group and so on).

- The study group includes 200 students who received the educational program about food safety and the control group includes 200 students who did not receive the related education program (gave them the same questionnaire in pre and post test).

\section{Program development stage}

Based on the information obtained from the base line assessment, in addition to literature, the researchers designed the educational program. The main aim of the program was to improve the students' knowledge and practice about food safety.

\section{Contents of the program}

- The program was designed by the researchers in a form of printed Arabic booklet. Preparation for the educational program was took about one month, it included the following:

* Definition of food safety

* Importance of food Safety

* Causes of food borne diseases

* Causes of food poisoning

* Symptoms of food poisoning

* Who are the most vulnerable to poisoning?

* How do we maintain the food safety?

- Personal hygiene

- Adequate food cooking

- preventing the transmission of infections and food poisoning.

- keeping food at safe temperatures

- Application of the program was carried out to the study group only.

- The program was discussed through two separate sessions; in the first session we discussed to all students, the definition of food safety, importance of food safety, causes of food borne diseases and cause of food poisoning, this session took about 1 hour (the first 10 minutes we communicated with the students, the body of the session took about 40 minutes and the last 10 minutes were open discussion). At the second day we discussed the second session which began with 10 minute revision the content of the first session, after that we took about 30-40 minutes to discuss the symptoms of food poisoning, who are the most vulnerable to poisoning?, how do we maintain the food safety? The last 20 minutes was to summarize the all contents of the program and open discussion.

-The methods of teaching used in program were lecturing followed by focused group discussions.

- A copy of the Arabic form of the program in form of Arabic booklet (include the details content of the program) was given to each student during the first session to read, review and remembers the content of the program at home. The booklet included pictures to demonstrate the content of the program.

\section{Evaluation stage}

- In order to assess the impact of the program, a posttest was done using the same tools.

-The students were asked the questions in the pre-test and they were asked again the same questions at the post-test.

- The post-test was conducted for both the study and control groups to assess the changes of knowledge and practices, and to find out to what extend students were following the instruction in the educational program (up to one month after completion the food safety educational program).

- The study participants completed the food safety questionnaire two times: pre-intervention (prior to food safety educational program), post-intervention (after the program).

- The mean score of food safety knowledge and practices were calculated for the study and control groups before and after conduction of educational program, then the students' mean score of knowledge and practices was compared before and after implementation of the educational program.

\section{Scoring}

- Answers to the questions related to food safety knowledge or practices were graded, one point being given for the right answer and zero for the wrong answer.

- The scores of the items were summed-up and then divided by the number of the items.

- The levels of the knowledge and practices was considered good if the percent score was more than $75 \%$, satisfactory if the percent score was between $50 \%-75 \%$ and poor if less than $50 \%$ (the total score of knowledge questions was 26 , and the total score practice questions was 31 ). 


\section{Ethical Considerations}

Informed consent was obtained orally from each student before full filling the questionnaire.

Confidentiality of the study participants were ascertained (as each student has the right to withdraw at any time and the data used only for the purpose of the study).

\section{Data analysis and reporting}

The collected data was coded, tabulated and analyzed by computer statistical programs (SPSS). Qualitative variables were discribed by number and percent where quantitative variables were discribed by mean and standard deviations,. Chai-square test used to compare qualitative variables where $\mathrm{t}$-test used to compare between quantitative variables. A two-tailed $\mathrm{p}<0.05$ was considered statistically significant. All analyses were performed with the SPSS 20.0 software.

\section{Results}

Table (1): Percentage distribution of the students and their families according to their socio-demographic characteristics $(\mathbf{N}=\mathbf{1 0 0 0})$.

\begin{tabular}{|c|c|c|}
\hline Item & No. & $\%$ \\
\hline \multicolumn{3}{|l|}{ A-students } \\
\hline \multicolumn{3}{|l|}{ Age in years } \\
\hline $15<16$ & 434 & 43.4 \\
\hline $17-18$ & 566 & 56.6 \\
\hline Mean age of students & $17.2 \pm 0.6$ & \\
\hline \multicolumn{3}{|l|}{ Gender } \\
\hline Male & 510 & 51.0 \\
\hline Female & 490 & 49.0 \\
\hline \multicolumn{3}{|l|}{ Frequency of Food Preparation by students } \\
\hline Daily & 190 & 19.0 \\
\hline Weekly & 730 & 73.0 \\
\hline Never & 80 & 8.0 \\
\hline \multicolumn{3}{|l|}{ Socio-economic status } \\
\hline Low & 210 & 21.0 \\
\hline Moderate & 620 & 62.0 \\
\hline High & 170 & 17.0 \\
\hline \multicolumn{3}{|l|}{ B-parents } \\
\hline \multicolumn{3}{|l|}{ Mother education } \\
\hline Informal (illiterate- read and write-primary) & 370 & 37.0 \\
\hline Formal (preparatory- secondary- university) & 630 & 63.0 \\
\hline \multicolumn{3}{|l|}{ Father education } \\
\hline Informal (illiterate- read and write-primary) & 290 & 29.0 \\
\hline Formal (preparatory- secondary- university) & 710 & 71.0 \\
\hline \multicolumn{3}{|l|}{ Mother occupation } \\
\hline Employed & 350 & 35.0 \\
\hline House wife & 650 & 65.0 \\
\hline
\end{tabular}


Table (2): Knowledge of adolescent students about food safety ( $n=1000)$.

\begin{tabular}{|l|c|c|c|c|c|}
\hline \multirow{2}{*}{ Items } & \multicolumn{2}{|c|}{ Correct answers } & \multicolumn{2}{c|}{ Incorrect answers } & \multirow{2}{*}{ Mean \pm SD } \\
\cline { 2 - 6 } & No. & $\%$ & No. & \% & \% \\
\hline $\begin{array}{l}\text { 1- General food safety knowledge (maximum } \\
\text { mean score=60.3) }\end{array}$ & 343 & 34.3 & 657 & 65.7 & $32.1 \pm 12.7$ \\
\hline $\begin{array}{l}\text { 2- knowledge about general and personal } \\
\text { hygiene } \\
\text { (maximum mean score=75) }\end{array}$ & 383 & 38.3 & 617 & 61.7 & $34.8 \pm 20.0$ \\
\hline $\begin{array}{l}\text { 3- knowledge about cooking food well (maximum } \\
\text { mean score=75) }\end{array}$ & 380 & 38 & 620 & 62 & $35.4 \pm 28.7$ \\
\hline $\begin{array}{l}\text { 4- knowledge about keeping food at safe } \\
\text { temperatures } \\
\text { (maximum mean score=60.0) }\end{array}$ & 268 & 26.8 & 732 & 73.2 & $25.3 \pm 24.9$ \\
\hline $\begin{array}{l}\text { 5- Knowledge about preventing the transmission } \\
\text { of infections and food poisoning. } \\
\text { (maximum mean score=49.3) }\end{array}$ & 240 & 24 & 760 & 76 & $27.8 \pm 13.5$ \\
\hline $\begin{array}{l}\text { Mean score of knowledge } \\
\text { (maximum mean score=56.8) }\end{array}$ & & & $37.4 \pm 8.9$ & \\
\hline
\end{tabular}

N.B: \# The subjects' response is more than one answer

Fig (1): Knowledge of adolescent students about food safety $(n=1000)$.

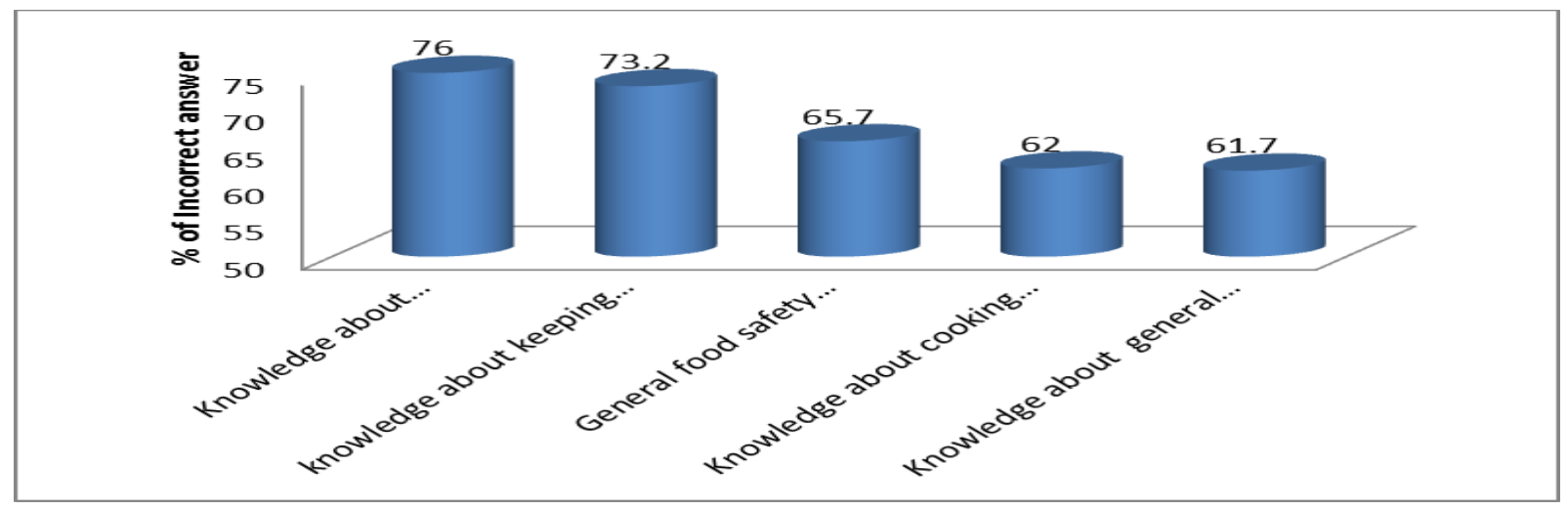

Table (3): Practice of adolescent students about food safety ( $n=1000)$.

\begin{tabular}{|l|c|c|c|c|c|}
\hline \multicolumn{1}{|c|}{ food safety practices } & \multicolumn{2}{c|}{ Correct answers } & \multicolumn{2}{|c|}{ Incorrect answers } & \multirow{2}{*}{ Mean \pm SD } \\
\cline { 2 - 6 } & No. & $\%$ & No. & \% & \\
\hline $\begin{array}{l}\text { 1- Practices about general and personal hygiene } \\
\text { (maximum mean score=54.8) }\end{array}$ & 345 & 34.5 & 655 & 65.5 & $31.8 \pm 18.0$ \\
\hline $\begin{array}{l}\text { 2- Practices about preventing the transmission of } \\
\text { infections and food poisoning } \\
\text { (maximum mean score=61.3) }\end{array}$ & 384 & 38.4 & 616 & 61.6 & $38.4 \pm 13.8$ \\
\hline $\begin{array}{l}\text { 3- Practices about keeping the food at safe } \\
\text { temperature (maximum mean score=52.2) }\end{array}$ & 233 & 23.3 & 767 & 76.7 & $22.0 \pm 22.2$ \\
\hline $\begin{array}{l}\text { 4-practices about cooking food well (maximum } \\
\text { mean score=60) }\end{array}$ & 305 & 30.5 & 695 & 69.5 & $39.0 \pm 23.6$ \\
\hline $\begin{array}{l}\text { Mean score of practices } \\
\text { (maximum mean score=49.7) }\end{array}$ & \multicolumn{4}{|l}{$35.6 \pm 7.7$} \\
\hline
\end{tabular}


Fig., (2): Practice of adolescent students about food safety $(n=1000)$.

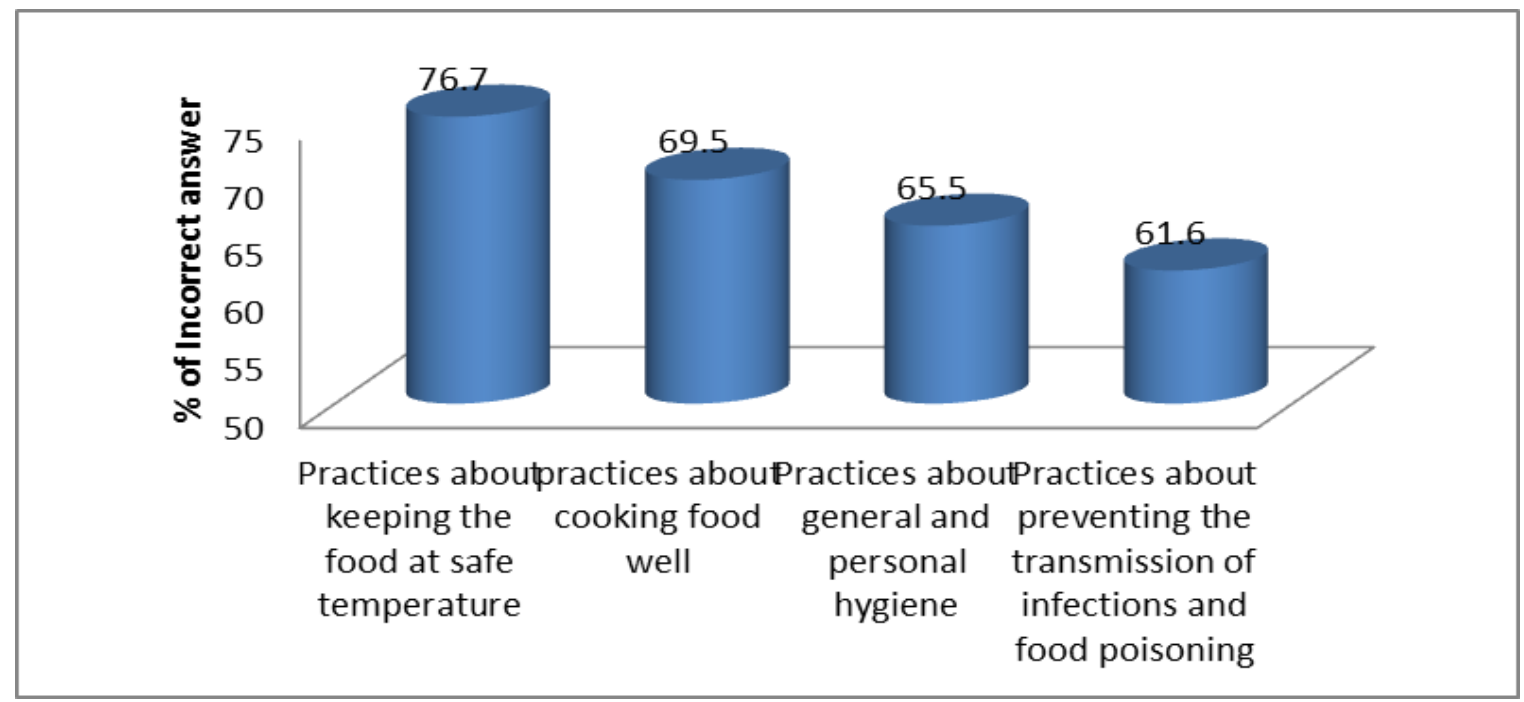

Fig (3): Students' levels of knowledge and practices regards the baseline assessment data about food safety $(\mathbf{n}=1000)$.

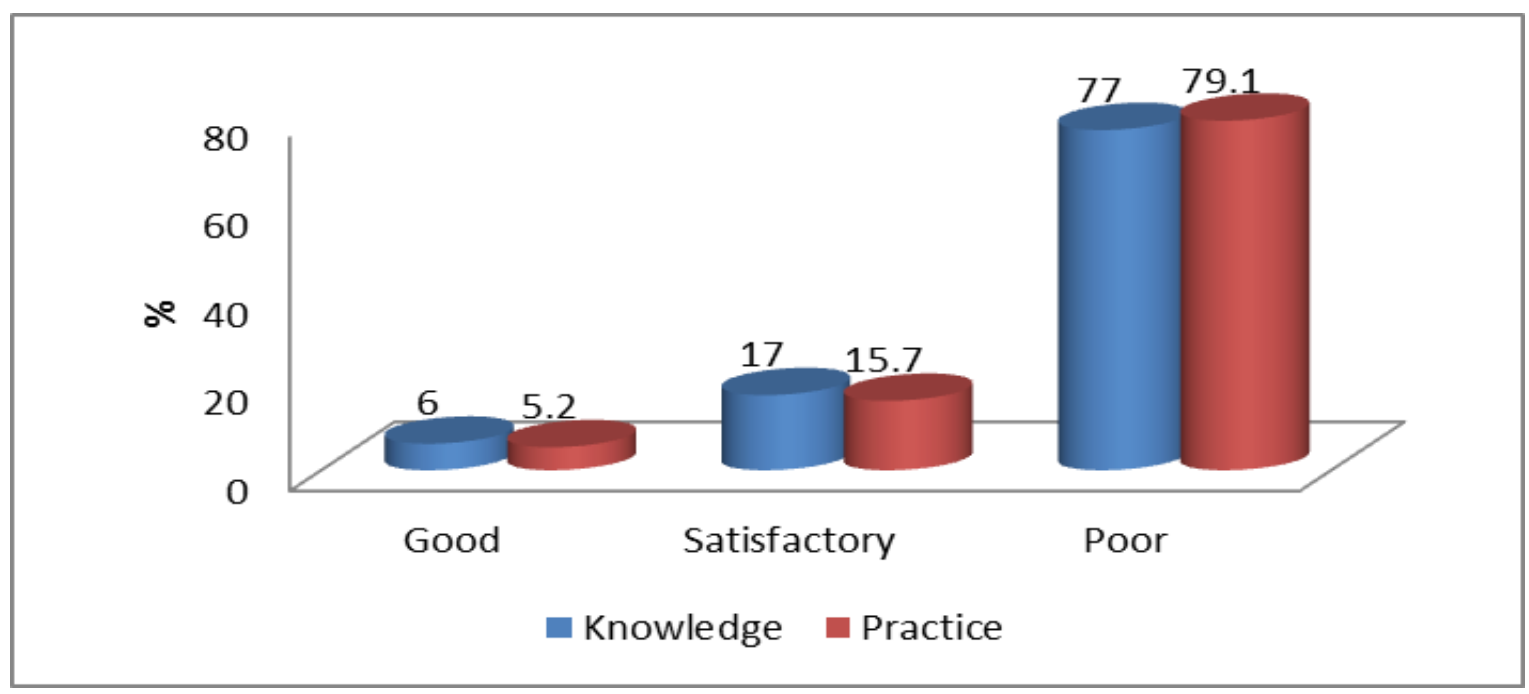

Table (4): The relation between students' mean score of knowledge and self-reported practices related to food safety and their socio-demographic characteristics $(N=1000)$.

\begin{tabular}{|c|c|c|c|c|c|c|}
\hline \multirow{2}{*}{ Items } & \multicolumn{3}{|c|}{ Knowledge (maximum score $=57.5$ ) } & \multicolumn{3}{|c|}{ Practice $($ maximum score $=59.4)$} \\
\hline & Mean & $\pm \mathrm{SD}$ & P.value & Mean & SD & P.value \\
\hline \multicolumn{7}{|l|}{ Gender } \\
\hline Male & \multicolumn{2}{|c|}{$29.9 \pm 8.8$} & \multirow{2}{*}{$0.001 * *$} & \multicolumn{2}{|c|}{$24.2 \pm 6.7$} & \multirow{2}{*}{$0.001 * *$} \\
\hline Female & \multicolumn{2}{|c|}{$38.4 \pm 9.4$} & & \multicolumn{2}{|c|}{$\mathbf{3 6 . 3} \pm 8.6$} & \\
\hline \multicolumn{7}{|l|}{ Age in years } \\
\hline$(15<16$ years $)$ & \multicolumn{2}{|c|}{$39.5 \pm 10.2$} & \multirow{2}{*}{0.157} & \multicolumn{2}{|c|}{$\frac{36.5 \pm 9.1}{35.1 \pm 0.5}$} & 015 \\
\hline (17-18 years) & \multicolumn{2}{|c|}{$37.9 \pm 10.2$} & & \multicolumn{2}{|c|}{$35.1 \pm 9.5$} & 0.215 \\
\hline \multicolumn{7}{|l|}{ Frequency of Food Preparation } \\
\hline Daily & \multicolumn{2}{|c|}{$\frac{1}{40.5 \pm 7.6}$} & \multirow{2}{*}{$0.001 * *$} & \multicolumn{2}{|c|}{$\mathbf{3 8 . 4} \pm 11.1$} & \multirow{2}{*}{$0.001 * *$} \\
\hline Weekly & \multicolumn{2}{|c|}{$33.8 \pm 10.9$} & & & & \\
\hline
\end{tabular}




\begin{tabular}{|c|c|c|c|c|c|c|}
\hline \multirow{2}{*}{ Items } & \multicolumn{3}{|c|}{ Knowledge (maximum score $=57.5$ ) } & \multicolumn{3}{|c|}{ Practice $($ maximum score $=59.4)$} \\
\hline & Mean & \pm SD & P.value & Mean & SD & P.value \\
\hline Never & \multicolumn{2}{|c|}{$26.5 \pm 8.0$} & & \multicolumn{2}{|c|}{$23.7 \pm 10.7$} & \\
\hline \multicolumn{7}{|l|}{ Socio-economic status level } \\
\hline Low & \multicolumn{2}{|c|}{$31.4 \pm 4.7$} & \multirow{3}{*}{$0.001 * *$} & \multicolumn{2}{|c|}{$28.1 \pm 9.0$} & \multirow{3}{*}{$0.001 * *$} \\
\hline Moderate & \multicolumn{2}{|c|}{$35.6 \pm 4.2$} & & \multicolumn{2}{|c|}{$31.8 \pm 12.3$} & \\
\hline High & \multicolumn{2}{|c|}{$39.5 \pm 5.7$} & & \multicolumn{2}{|c|}{$41.4 \pm 6.8$} & \\
\hline Mother education & & & & & & \\
\hline Informal & \multicolumn{2}{|c|}{$33.0 \pm 10.4$} & \multirow{2}{*}{$0.010 * *$} & \multicolumn{2}{|c|}{$28.3 \pm 10.2$} & \multirow{2}{*}{$0.001 * *$} \\
\hline Formal & & & & & & \\
\hline \multicolumn{7}{|l|}{ Father education } \\
\hline Informal & \multirow{2}{*}{\multicolumn{2}{|c|}{$\begin{array}{c}34.7 \pm 10.8 \\
\mathbf{3 7 . 3} \pm 9.5\end{array}$}} & \multirow{2}{*}{$0.013 *$} & \multirow{2}{*}{\multicolumn{2}{|c|}{$\begin{array}{c}30.6 \pm 11.7 \\
\mathbf{3 6 . 5} \pm 9.1\end{array}$}} & \multirow{2}{*}{$0.001 * *$} \\
\hline Formal & & & & & & \\
\hline \multicolumn{7}{|l|}{ Mother occupation } \\
\hline Employed & \multicolumn{2}{|c|}{$39.1 \pm 10.4$} & \multirow{2}{*}{$0.017 *$} & \multirow{2}{*}{\multicolumn{2}{|c|}{$\begin{array}{l}36.6 \pm 10.9 \\
33.1+118\end{array}$}} & \multirow{2}{*}{$0.012 *$} \\
\hline House wife & \multicolumn{2}{|c|}{$35.1 \pm 10.5$} & & & & \\
\hline
\end{tabular}

*Statistically Significant differences $\quad * *$ Highly Statistically Significant differences

Table (5): Percentage distribution of socio-demographic characteristics of students in the study and the control groups.

\begin{tabular}{|c|c|c|c|c|c|}
\hline \multirow{2}{*}{ Items } & \multicolumn{2}{|c|}{ Control $(\mathrm{N}=200)$} & \multicolumn{2}{|c|}{ Study $(\mathrm{N}=200)$} & \multirow{2}{*}{ P. value } \\
\hline & No. & $\%$ & No. & $\%$ & \\
\hline \multicolumn{6}{|l|}{ Gender } \\
\hline Male & 102 & 51.0 & 94 & 47.0 & \multirow{2}{*}{0.484} \\
\hline Female & 98 & 49.0 & 106 & 53.0 & \\
\hline \multicolumn{6}{|l|}{ Age } \\
\hline (15<16 years) & 88 & 44.0 & 92 & 46.0 & \multirow{2}{*}{0.687} \\
\hline (17-18 years) & 112 & 56.0 & 108 & 54.0 & \\
\hline \multicolumn{6}{|c|}{ Frequency of Food Preparation } \\
\hline Daily & 38 & 19.0 & 48 & 24.0 & \multirow{3}{*}{0.468} \\
\hline Weekly & 146 & 73.0 & 136 & 68.0 & \\
\hline Never & 16 & 8.0 & 16 & 8.0 & \\
\hline \multicolumn{6}{|l|}{ Socio-economic status } \\
\hline Low & 42 & 21.0 & 36 & 18.0 & \multirow{3}{*}{0.517} \\
\hline Moderate & 124 & 62.0 & 122 & 61.0 & \\
\hline High & 34 & 17.0 & 42 & 21.0 & \\
\hline \multicolumn{6}{|l|}{ Mother education } \\
\hline Informal & 74 & 37.0 & 72 & 36.0 & \multirow{2}{*}{0.835} \\
\hline Formal & 126 & 63.0 & 128 & 64.0 & \\
\hline \multicolumn{6}{|l|}{ Father education } \\
\hline Informal & 58 & 29.0 & 56 & 28.0 & \multirow[b]{2}{*}{0.825} \\
\hline Formal & 142 & 71.0 & 144 & 72.0 & \\
\hline \multicolumn{6}{|l|}{ Mother occupation } \\
\hline Employed & 60 & 30.0 & 64 & 32.0 & \multirow{2}{*}{0.378} \\
\hline House wife & 140 & 70.0 & 136 & 68.0 & \\
\hline
\end{tabular}


Table (6): The relation between mean score of knowledge among students in the study and control groups.

\begin{tabular}{|c|c|c|c|c|c|c|c|}
\hline \multirow{2}{*}{ Mean score of knowledge } & \multicolumn{2}{|c|}{$\begin{array}{c}\text { Control }(\mathrm{N}=200) \\
(\text { mean }+\mathrm{SD})\end{array}$} & \multirow[t]{2}{*}{$\mathbf{P 1}$} & \multicolumn{2}{|c|}{$\begin{array}{c}\text { Study }(\mathrm{N}=200) \\
(\text { mean }+ \text { SD })\end{array}$} & \multirow[t]{2}{*}{$\mathbf{P 2}$} & \multirow{2}{*}{$\begin{array}{l}\text { P3 } \\
\text { P4 }\end{array}$} \\
\hline & Pre-test & Post-test & & Pre-test & Post-test & & \\
\hline $\begin{array}{l}\text { 1- General Information } \\
\text { about food safety } \\
(\text { maximum score }=100)\end{array}$ & $28.4 \pm 12.7$ & $30.6 \pm 13.4$ & 0.094 & $30.8 \pm 12.2$ & $93.4 \pm 14.2$ & $<0.001 * *$ & $\begin{aligned} \mathbf{P 3} & =0.055 \\
\mathbf{P 4} & <0.001 * *\end{aligned}$ \\
\hline $\begin{array}{l}\text { 2- knowledge about } \\
\text { personal hygiene and } \\
\text { general cleanliness of food } \\
\text { safety (maximum } \\
\text { score }=100 \text { ) }\end{array}$ & $34.8 \pm 20$ & $36.3 \pm 19.2$ & 0.445 & $33.5 \pm 20.1$ & $93 \pm 17$ & $<0.001 * *$ & $\begin{aligned} \mathbf{P 3} & =0.534 \\
\mathbf{P 4} & <0.001 * *\end{aligned}$ \\
\hline $\begin{array}{l}\text { 3- Students' knowledge } \\
\text { about cooking good food } \\
\text { (maximum score }=100)\end{array}$ & $32 \pm 28.7$ & $35.3 \pm 26.7$ & 0.231 & $35 \pm 26.9$ & $93.7 \pm 15.5$ & $<0.001^{* *}$ & $\begin{aligned} \mathbf{P 3} & =0.281 \\
\mathbf{P 4} & <0.001 * *\end{aligned}$ \\
\hline $\begin{array}{l}\text { 4- knowledge about } \\
\text { keeping food at safe } \\
\text { temperatures (maximum } \\
\text { score=100) }\end{array}$ & $25.3 \pm 24.9$ & $28.3 \pm 23.7$ & 0.218 & $25.8 \pm 24.2$ & $92.3 \pm 17.6$ & $<0.001 * *$ & $\begin{array}{c}\mathbf{P 3}=0.839 \\
\mathbf{P 4}<0.001 * *\end{array}$ \\
\hline $\begin{array}{l}\text { 5- knowledge for } \\
\text { preventing transmission of } \\
\text { food poisoning (maximum } \\
\text { score }=100 \text { ) }\end{array}$ & $47.3 \pm 13.5$ & $47.9 \pm 13.9$ & 0.678 & $49.6 \pm 13.2$ & $94.7 \pm 14.1$ & $<0.001 * *$ & $\begin{array}{c}\mathbf{P 3}=0.088 \\
\mathbf{P 4}<0.001 * *\end{array}$ \\
\hline $\begin{array}{l}P 1=\text { between pre-test and p } \\
P 3=\text { between control and stu } \\
* \text { Statistically Significant diffe }\end{array}$ & $\begin{array}{l}\text {-test in } \\
\text { in pre- } \\
\text { ices }\end{array}$ & rol & & $\begin{array}{l}P 2=b e \\
P 4=b e \\
\text { Highly }\end{array}$ & $\begin{array}{l}\text { en pre-tes } \\
\text { en control } \\
\text { stically } \mathrm{Si}\end{array}$ & $\begin{array}{l}\text { Ind post-t } \\
\text { d study in } \\
\text { ficant dif. }\end{array}$ & $\begin{array}{l}\text { t in study } \\
\text { ost-test } \\
\text { rences }\end{array}$ \\
\hline
\end{tabular}

Table (7): The relation between mean score of practice among students in the study and control groups.

\begin{tabular}{|c|c|c|c|c|c|c|c|}
\hline \multirow[t]{2}{*}{ Mean score of practices } & \multicolumn{2}{|c|}{$\begin{array}{c}\text { Control }(\mathrm{N}=200) \\
(\text { mean }+\mathrm{SD})\end{array}$} & \multirow[t]{2}{*}{ P1 } & \multicolumn{2}{|c|}{$\begin{array}{r}\text { Study }(\mathrm{N}=200) \\
(\text { mean } \pm \text { SD })\end{array}$} & \multirow[t]{2}{*}{$\mathbf{P 2}$} & \multirow{2}{*}{$\begin{array}{l}\text { P3 } \\
\text { P4 }\end{array}$} \\
\hline & Pre-test & Post-test & & Pre-test & Post-test & & \\
\hline $\begin{array}{l}\text { 1-Personal hygiene } \\
\text { Practices } \\
(\text { maximum score }=100)\end{array}$ & $31.8 \pm 18.1$ & $33.4 \pm 19.2$ & 0.408 & $33.5 \pm 19.2$ & $93.5 \pm 14.1$ & $<0.001 * *$ & $\begin{aligned} \mathbf{P 3} & =0.355 \\
\mathbf{P 4} & =<0.001 * *\end{aligned}$ \\
\hline $\begin{array}{l}\text { 2-Cross Contamination } \\
\text { Practices } \\
(\text { maximum score }=100)\end{array}$ & $38.4 \pm 13.9$ & $40.8 \pm 16.1$ & 0.115 & $38.4 \pm 14$ & $93.9 \pm 14.6$ & $<0.001 * *$ & $\begin{aligned} \mathbf{P 3} & =0.998 \\
\mathbf{P 4} & =<0.001 * *\end{aligned}$ \\
\hline $\begin{array}{l}\text { 3-Time temperature } \\
\text { Practices } \\
(\text { maximum score }=100)\end{array}$ & $22 \pm 22.2$ & $24.8 \pm 23.1$ & 0.226 & $\begin{array}{l}25.3 \pm \\
21.7\end{array}$ & $93 \pm 15.5$ & $<0.001 * *$ & $\begin{array}{c}\mathbf{P 3}=0.141 \\
\mathbf{P 4}=<0.001^{*} *\end{array}$ \\
\hline $\begin{array}{l}\text { 4-Cooking foods } \\
\text { adequately } \\
\text { practices } \\
\text { (maximum score }=100 \text { ) }\end{array}$ & $39 \pm 23.7$ & $39.3 \pm 25.2$ & 0.892 & $41.7 \pm 21.3$ & $94.7 \pm 14.7$ & $<0.001 * *$ & $\begin{aligned} \mathbf{P 3} & =0.237 \\
\mathbf{P 4} & =<0.001 * *\end{aligned}$ \\
\hline
\end{tabular}

$P 1=$ between pre-test and post-test in control

P3 = between control and study in pre-test

**Highly Statistically Significant differences
$P 2=$ between pre-test and post-test in study

$P 4=$ between control and study in post-test 
Table (8): Comparison between the mean score of pre- post intervention of knowledge related to food safety among students in the control and the study groups and their socio-demographic data.

\begin{tabular}{|c|c|c|c|c|c|c|}
\hline \multirow{3}{*}{ Items } & \multicolumn{2}{|c|}{$\begin{array}{c}\text { Pre knowledge }(\mathbf{N}=\mathbf{2 0 0}) \\
(\text { Maximum score }=56.8)\end{array}$} & \multirow{3}{*}{ P. value } & \multicolumn{2}{|c|}{$\begin{array}{l}\text { Post knowledge }(\mathbf{N}=\mathbf{2 0 0}) \\
\text { (Maximum score }=100)\end{array}$} & \multirow{3}{*}{ P. value } \\
\hline & Control( $(N=200)$ & Study $(N=200)$ & & $\operatorname{Control}(\mathrm{N}=\mathbf{2 0 0})$ & $\operatorname{Study}(\mathrm{N}=200)$ & \\
\hline & Mean \pm SD & Mean \pm SD & & Mean \pm SD & Mean \pm SD & \\
\hline \multicolumn{7}{|l|}{ Age } \\
\hline$(15<16$ years $)$ & $39.5 \pm 10.3$ & $38.4 \pm 10.4$ & 0.576 & $40.1+9.4$ & $83.7 \pm 23$ & $<0.001 * *$ \\
\hline (17-18 years) & $33.9 \pm 10.3$ & $36+9.5$ & 0.075 & $36.2 \pm 9.4$ & $97.4+3.7$ & $<0.001 * *$ \\
\hline \multicolumn{7}{|c|}{ Frequency of Food Preparation } \\
\hline Daily & $40.5 \pm 7.7$ & $39.3 \pm 9.1$ & 0.522 & $41.2 \pm 7.3$ & $91.7 \pm 13.1$ & $<0.001 * *$ \\
\hline Weekly & $35.3 \pm 8.2$ & $35.9 \pm 5.4$ & 0.821 & $36.4 \pm 9.2$ & $98.4 \pm 3.1$ & $<0.001 * *$ \\
\hline Never & $33.8 \pm 10.9$ & $35.8 \pm 10.3$ & 0.109 & $36 \pm 9.8$ & $93.7 \pm 14.9$ & $<0.001 * *$ \\
\hline \multicolumn{7}{|c|}{ Socio-economic status } \\
\hline High & $37.1 \pm 9.3$ & $37.5 \pm 9$ & 0.779 & $37.3 \pm 7.4$ & $97.3 \pm 4.2$ & $<0.001 * *$ \\
\hline Moderate & $35.8 \pm 5.8$ & $36.5 \pm 9$ & 0.350 & $36.9 \pm 10.3$ & $94 \pm 13.6$ & $<0.001 * *$ \\
\hline Low & $34.4 \pm 11.8$ & $36.4 \pm 10.4$ & 0.146 & $37.3 \pm 9$ & $87.7 \pm 19.7$ & $<0.001 * *$ \\
\hline \multicolumn{7}{|c|}{ Mother education } \\
\hline Informal & $32.9 \pm 10.5$ & $35.2 \pm 10.1$ & 0.082 & $35.8 \pm 9.6$ & $97.1+5.8$ & $<0.001 * *$ \\
\hline Formal & $39.1 \pm 9.3$ & $39.3 \pm 8.9$ & 0.936 & $39.3 \pm 9.1$ & $87.3 \pm 20.5$ & $<0.001 * *$ \\
\hline \multicolumn{7}{|c|}{ Father education } \\
\hline Informal & $34.7 \pm 10.9$ & $36.6 \pm 10.2$ & 0.129 & $37 \pm 9.9$ & $95.7 \pm 9.8$ & $<0.001 * *$ \\
\hline Formal & $36.3 \pm 9.5$ & $36.7 \pm 8.9$ & 0.794 & $37.2 \pm 8.6$ & $88.5 \pm 20$ & $<0.001 * *$ \\
\hline \multicolumn{7}{|c|}{ Mother occupation } \\
\hline House wife & $35.1 \pm 10.5$ & $36.3 \pm 9.8$ & 0.532 & $37 \pm 9.6$ & $93.9 \pm 13.9$ & $<0.001 * *$ \\
\hline Employed & $39.1 \pm 10$ & $42.6 \pm 8.9$ & 0.228 & $39.1 \pm 10$ & $87.9 \pm 14.9$ & $<0.001 * *$ \\
\hline
\end{tabular}

**Highly Statistically Significant differences

Table (9):_Comparison between the mean score of pre- post intervention of self-reported practices related to food safety among students in the control and the study groups and their socio-demographic data.

\begin{tabular}{|c|c|c|c|c|c|c|}
\hline \multirow{3}{*}{ Socio-demographic data } & \multicolumn{2}{|c|}{$\begin{array}{c}\text { Pre practice }(\mathrm{N}=200) \\
(\text { Maximum score }=49.7)\end{array}$} & \multirow{3}{*}{ P. value } & \multicolumn{2}{|c|}{$\begin{array}{l}\text { Post practice }(\mathrm{N}=200) \\
(\text { Maximum score }=100)\end{array}$} & \multirow{3}{*}{ P. value } \\
\hline & Control(N=200) & $\operatorname{Study}(N=200)$ & & Control(N=200) & $\operatorname{Study}(N=200)$ & \\
\hline & Mean \pm SD & Mean \pm SD & & Mean + SD & Mean \pm SD & \\
\hline \multicolumn{7}{|l|}{ Age } \\
\hline$(15<16$ years $)$ & $36.5 \pm 9.1$ & $38.5 \pm 9.7$ & 0.315 & $38.3 \pm 10.9$ & $85.6 \pm 22.6$ & $<0.001 * *$ \\
\hline (17-18 years) & $32.2 \pm 12.2$ & $33.2 \pm 11.8$ & 0.462 & $34.1 \pm 13.2$ & $96.9 \pm 3.6$ & $<0.001 * *$ \\
\hline \multicolumn{7}{|c|}{ Frequency of Food Preparation } \\
\hline Daily & $36 \pm 11.2$ & $37.3 \pm 11.2$ & 0.594 & $38.5 \pm 12.3$ & $93.4 \pm 12.1$ & $<0.001 * *$ \\
\hline Weekly & $32.8+11.9$ & $34+11.7$ & 0.387 & $34.6+13$ & $93.5+14.4$ & $<0.001 * *$ \\
\hline Never & $29.8 \pm 11$ & $32.7 \pm 8.9$ & 0.421 & $30.8 \pm 11.4$ & $96.7 \pm 3.1$ & $<0.001 * *$ \\
\hline \multicolumn{7}{|l|}{ Socio-economic status } \\
\hline High & $41.4+6.9$ & $42.8+7.6$ & 0.113 & $43.4+7.8$ & $97.1 \pm 3.1$ & $<0.001 * *$ \\
\hline Moderate & $31.8 \pm 12.4$ & $33.5 \pm 12.3$ & 0.275 & $33.5 \pm 13.1$ & $94.5 \pm 12.8$ & $<0.001 * *$ \\
\hline Low & $28.1 \pm 9.2$ & $31.3 \pm 8.5$ & 0.414 & $30.1 \pm 12.5$ & $87.2 \pm 19.2$ & $<0.001 * *$ \\
\hline
\end{tabular}




\begin{tabular}{|c|c|c|c|c|c|c|}
\hline \multirow{3}{*}{ Socio-demographic data } & \multicolumn{2}{|c|}{$\begin{array}{c}\text { Pre practice }(\mathrm{N}=200) \\
(\text { Maximum score }=49.7)\end{array}$} & \multirow{3}{*}{ P. value } & \multicolumn{2}{|c|}{$\begin{array}{l}\text { Post practice }(\mathrm{N}=200) \\
(\text { Maximum score }=100)\end{array}$} & \multirow{3}{*}{ P. value } \\
\hline & $\operatorname{Control}(\mathrm{N}=200)$ & $\operatorname{Study}(N=200)$ & & Control(N=200) & $\operatorname{Study}(\mathbf{N}=200)$ & \\
\hline & Mean \pm SD & Mean \pm SD & & Mean + SD & Mean \pm SD & \\
\hline \multicolumn{7}{|l|}{ Mother education } \\
\hline Informal & $28.2 \pm 10.2$ & $30.1 \pm 10.3$ & 0.134 & $30 \pm 12.2$ & $88.7 \pm 19.9$ & $<0.001 * *$ \\
\hline Formal & $42+8.8$ & $42.8 \pm 8.7$ & 0.558 & $43.8 \pm 8.7$ & $96.5 \pm 5.7$ & $<0.001 * *$ \\
\hline \multicolumn{7}{|l|}{ Father education } \\
\hline Informal & $30.6 \pm 11.7$ & $32.4 \pm 11.3$ & 0.180 & $32.4 \pm 13.1$ & $89 \pm 19$ & $<0.001 * *$ \\
\hline Formal & $39.5 \pm 9.2$ & $40.3 \pm 9.8$ & 0.651 & $41.4 \pm 9.5$ & $95.6 \pm 9.5$ & $<0.001 * *$ \\
\hline \multicolumn{7}{|l|}{ Mother occupation } \\
\hline House wife & $33.1 \pm 11.9$ & $34.7 \pm 11.7$ & 0.758 & $34.9 \pm 13$ & $94 \pm 13.2$ & $<0.001 * *$ \\
\hline Employed & $36.6 \pm 2.3$ & $35.4 \pm 7$ & 0.191 & $38.5 \pm 0$ & $87.7 \pm 14.2$ & $<0.001 * *$ \\
\hline
\end{tabular}

**Highly Statistically Significant differences

Fig (4): Comparison between students in the study and the control groups according to their mean score of knowledge and practice in the pre- post intervention.

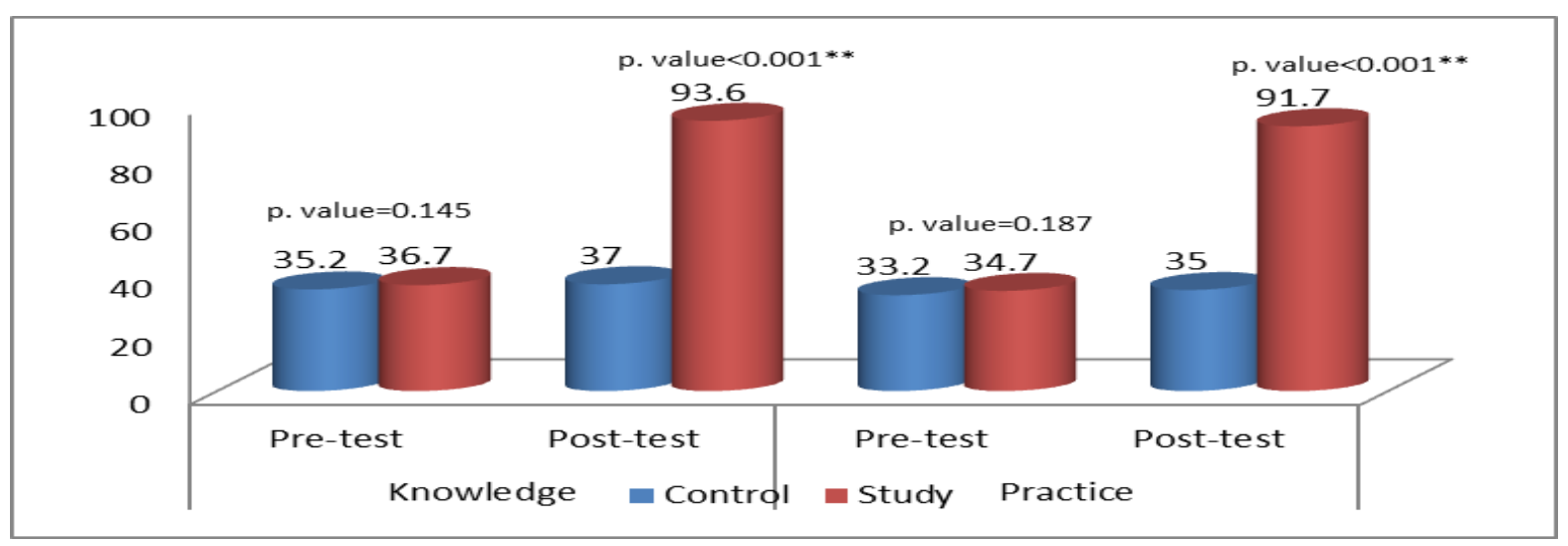

Fig (5): The score of knowledge in the pre and the post- test among students in the study and the control groups

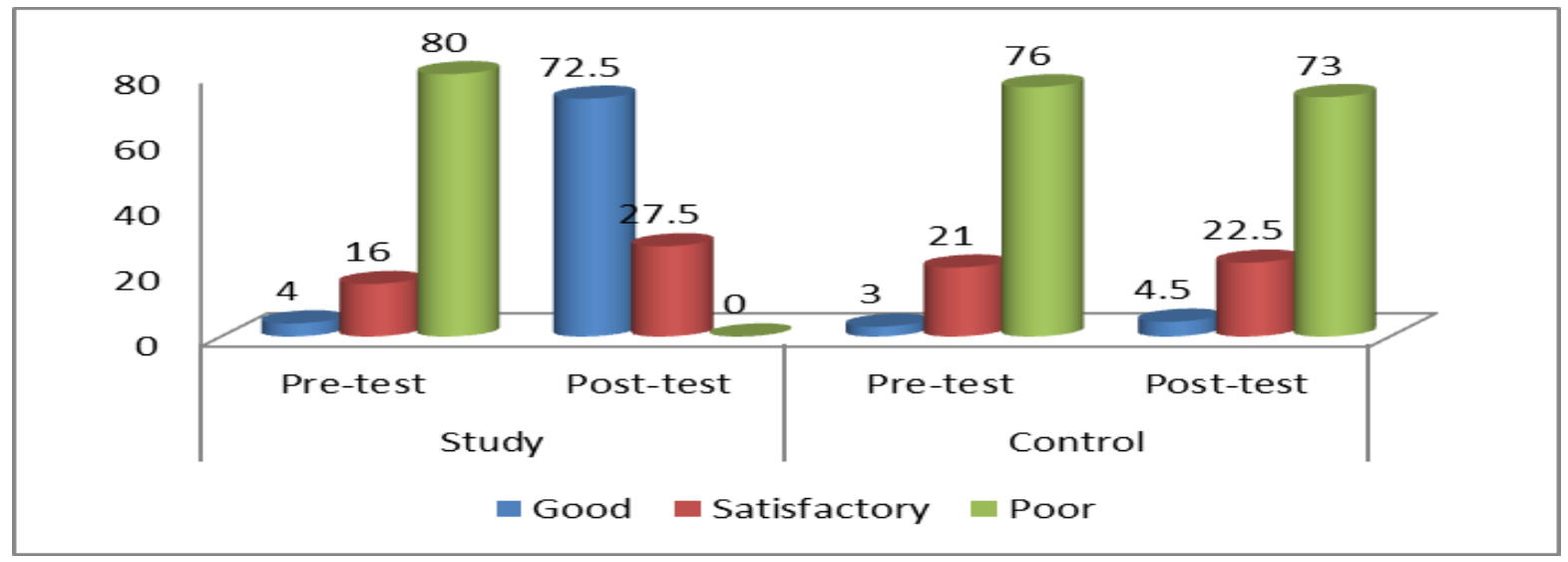


Fig (6): The score of self-reported practices in the pre and the post- test among students in the study and the control groups.

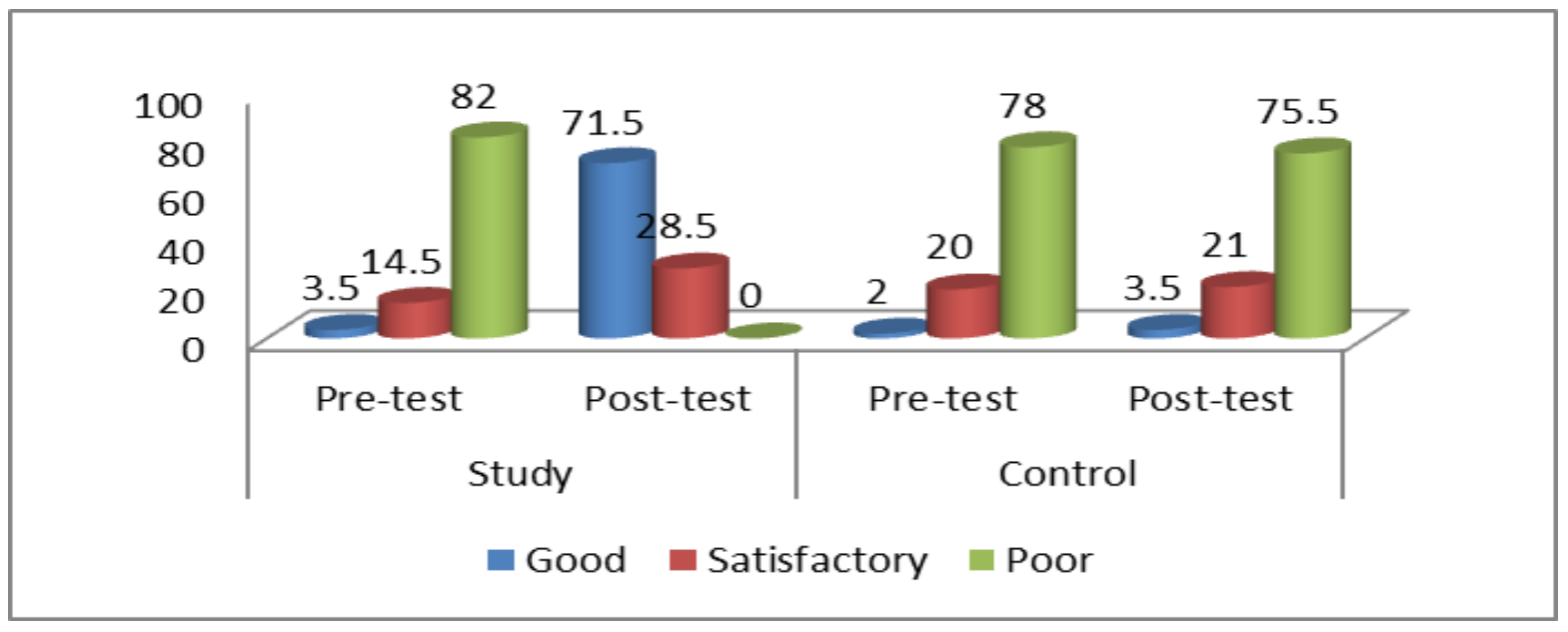

Table (1) : illustrates the socio-demographic characteristics of students. Male and female students constituted $(51 \%)$ and $(49 \%)$ respectively. More than half of the respondents were between the ages of 1718 years $(56.6 \%)$ with a mean age of $(17.2 \pm 0.6)$. Only $(8 \%)$ of the respondents indicated that they never prepared food, (19\%) prepared food daily, while nearly three quarter $(73 \%)$ of them prepared food weekly. More than two thirds of mothers and fathers had formal education (63\%) and (71\%) respectively. More than two thirds of the mothers were housewife $(65 \%)$.

Table (2) : illustrates the knowledge of adolescent students about food safety. The knowledge questions were categorized into five major contributors to food borne illness. Concerning right answers the table clarified that only (24\%) of the students answered correctly the questions related to preventing the transmission of infections and food poisoning with mean score of knowledge $(27.8 \pm 13.5)$ out of 7 items, $(38.3 \%)$ of the students answered correctly the questions related to general and personal hygiene with mean score of knowledge $(34.8 \pm 20.0)$ out of 4 items, followed by the knowledge about cooking foods adequately (38\%) with mean score of knowledge $(35.4 \pm 28.7)$ out of 4 items, followed by the knowledge about general food safety knowledge $(34.3 \%)$ with mean score of knowledge $(32.1 \pm 12.7)$ out of 6 items, followed by the knowledge about keeping foods at safe temperatures (26.8\%) with mean score of knowledge $(25.3 \pm 24.9)$ out of 5 items. The over Mean score of knowledge all was 37.4+8.9 out of 26 items. Regarding incorrect answers

figure (1) : revealed that, more than three fourths_of students $(76 \%)$ answered incorrectly the questions related to preventing the transmission of infections and food poisoning, knowledge about keeping food at safe temperatures $(73.2 \%)$, followed by general food safety knowledge $(65.7 \%)$, followed by the knowledge about cooking foods well (62\%), followed by general and personal hygiene knowledge $(61.7 \%)$.

Table (3) : presents the practice of adolescent students about food safety. The practices questions were categorized into four major contributors to food borne illness. Only (23.3\%) of the students answered correctly the questions related to keeping foods at safe temperatures with mean score of practices (22.0 \pm 22.2 ) out of 7 items, followed by the practices about cooking foods adequately (30.5\%) with mean score of practices $(39.0 \pm 23.6)$ out of 5 items, followed by the practices about the questions related to general and personal hygiene (34.5\%) with mean score of practices $(31.8 \pm 18.0)$ out of 11 items, and finally followed by the practices about preventing the transmission of infections and food poisoning $(38.4 \%)$ with mean score of practices $(38.4 \pm 13.8)$ out of 8 items. The overall Mean score of practices was $(35.6+7.7)$ out of 31 items. Regarding incorrect answers figure (2) : revealed that, more than three fourths $(76.7 \%)$ of students answered incorrectly the questions related to keeping foods at safe temperatures, followed by practices about cooking foods adequately (69.5\%), followed by answered incorrectly the questions related to practices about general and personal hygiene $(65.5 \%)$, and finally followed by practices related to preventing the transmission of infections and food poisoning $(61.6 \%)$.

Figure (3) : illustrates the students' levels of knowledge and practices regarding the baseline assessment data about food safety. As illustrated in this figure it was clear that, more than three fourth of students had poor knowledge and practices related to 
food safety (77\%) and (79.1\%) respectively and only $(6 \%)$ and $(5.2 \%)$ of them had good knowledge and practices respectively.

Table (4) : illustrates the relation between students' mean score of knowledge and practices related to food safety and their socio-demographic characteristics. Statistical significant differences were found between the mean score of students' knowledge and practices related to food safety and their gender and frequency of food preparation with high prevalence of knowledge and practices among the female students and students who prepare the food daily $(\mathrm{P}=0.001 \mathrm{per}$ each item $)$. No Statistical significant differences were found between the mean score of knowledge and practices with the students' age.

Statistical significant differences were found between the mean score of students' knowledge and practices related to food safety and their' socio-economic status with high prevalence of knowledge and practices among students of high socio-economic level. Also statistical significant differences were found between the mean score of students' knowledge and practices related food safety and their mothers' education, fathers' education, and mothers' occupations with high prevalence of knowledge and practices among students of mothers' and fathers' with formal education, and employed mothers $(\mathrm{P}=0.001$ per each item).

There was no statistically significant difference between demographic characteristics of students in the control and the study groups as illustrated in Table (5).

No statistical significant differences were found between knowledge score related to safety food of the pre and post test among students in the control group, and between knowledge of students in the control and the study groups in the pre- test. It was noticed that there were statistical significant differences between the pre and post test knowledge among students in study group and between knowledge score of students in the control and the study groups in the post-test $\mathrm{P}<0.001$ as clarified in Table (6).

Table (7) : Shows that, no statistical significant differences between the mean score of self- reported practices related to food safety in the pre-test and the post-test among students in the control group and between self- reported practices related to food safety among students in the control and study groups in pre-test regarding their personal hygiene, cross Contamination, time temperature, cooking foods adequately practices, $\mathrm{p}=<0.001$.

Statistical significant differences were found between the mean score of self- reported practices related to food safety in the pre-test and post-test among students in the study group and also between students in the control and the study groups in the post-test, $\mathrm{p}$ $=<0.001$.

Table (8) : shows that, no statistical significant differences were observed between mean score of knowledge in the pre -test among students in the study and the control groups and their gender, age, frequency of Food Preparation, Socio-economic status, mothers' and fathers' education, and mothers' occupation.

Statistical significant differences were found between mean score of knowledge in the post -test among students in the study and the control groups with high prevalence knowledge among male students, students at the age 17-18, students who prepare the food weekly, of high socio-economic families, mothers' and fathers' with informal education, employed mothers, and housewife mothers, $\mathrm{p}=<0.001$.

Table (9) : shows that, no statistical significant differences were found between mean score of selfreported practices in the pre -test among students in the study and the control groups and their gender, age, frequency of Food Preparation, Socio-economic status, mothers' and fathers' education, and mothers' occupation.

Statistical significant differences were found between mean score of self-reported practices in the post -test among students in the study and the control groups with high prevalence self-reported among female students, students at the age 17-18, students who never prepare the food, of high socio-economic families, mothers' and fathers' with informal education, employed mothers, and housewife mothers, $\mathrm{p}=<0.001$.

Figure (4) : illustrates comparison between students in the study and the control groups according to their mean score of knowledge and practice in the prepost intervention. A higher increasing of the post intervention knowledge and practices among students in the study group than those in the control group related to food safety. (Knowledge 37, 93.6\&practices $35,91.7$ for the control and the study groups respectively)

Fig (5) : shows the levels of knowledge in the pre and the post- test among students in the study and the control groups. Majority of students in the study group $(80 \%)$ had poor score of knowledge and only $(4 \%)$ had good score in the pre-test, but at the posttest $72 \%$ of them had got good score of knowledge and $27.5 \%$ of them had satisfactory score of knowledge.

As regard the control group in the pre- test, more than three fourth percent of students $(76 \%)$ had poor score of knowledge and only (3\%) of them has good score, as well as $73 \%$ of them had got poor score of knowledge in the post test and only $(4.5 \%)$ had good score. 
Fig (4) shows the levels of self-reported practices in the pre and the post- test among students in the study and the control groups, as regards the study group, the majority of students $(82 \%)$ had got poor score of practices in the pre test and only $(3.5 \%)$ of them had good score, but at the post- test $71.5 \%$ of them had got good score of practices and $(28.5 \%)$ of them had satisfactory score. As for the control group in the pretest more than three fourth of students $(78 \%)$ had got poor score of practices and only $(2 \%)$ of them had good score, also $(75.5 \%)$ of them had got poor score of practices in the post test and only (3.5\%) of them had good score.

\section{Discussion}

Food safety is an increasingly important public health issue since years ago until now. Increasingly cases that involved on consume unhealthy food becoming more severe, not only implicate the public, but more worrying rise among school children (Norazmir et al., 2012). The previous studies have demonstrated that food safety knowledge among secondary and tertiary students is limited (Jevsnik et al., 2008), therefore this work has been carried out to investigate the impact of educational program about food safety on improving adolescent students' knowledge and practices.

The base line assessment of food safety results in current study indicated that the adolescent students had limited knowledge of food safety and practices that put them at risk for food-borne illness. Lower knowledge scores are likely related to a lack of exposure to food safety education and opportunities to learn and practice safe food handling practices. This finding was consistent with previous studies carried out by (McArthur et al., 2007) and (Bramlett, (2011).

In the present study, males' students were (51\%) while the females' students were $(49 \%)$. More than three fourth of the students were between the ages 17-18 years, nearly three fourth of them prepared food weekly. More than two thirds of mothers and fathers had formal education. More than two thirds $(65.0 \%)$ of the mothers were housewife. Only (27\%) of families' income/month was more than 1500 pounds. These findings were near a by another findings conducted by (Yasemin, et al., (2013) who stated that, percentages of male and female respondents were about (50.6\%) and (49.4\%) respectively more than half of respondents were aged 18 of a total of $(50.4 \%)$ and followed by those who aged 16; around $(36.6 \%)$. Results also showed that, percentage of respondent in low-income family and high income family is same about (37\%) while only $(25.9 \%)$ of respondent came from moderate family.

In the current study, low percent of the students answered correctly the questions related to preventing the transmission of infections and food poisoning, $(38.3 \%)$ of the students answered correctly the questions related to general and personal hygiene, followed by the knowledge about cooking foods adequately, followed by the knowledge about general food safety knowledge, followed by the knowledge about keeping foods at safe temperatures. These findings are similar to many studies, which found that students' scores were very low (Curtis, 2008). Also same as the results of (Angolo, 2011) who stated that, the majority of respondents had low scores on questions related to cooking foods adequately, keeping foods at safe temperatures, and risks for food contamination.

The present study depicted that, low percent of the students answered correctly the questions related to keeping foods at safe temperatures, followed by the practices about cooking foods adequately, followed by the practices about the questions related to general and personal hygiene, and finally followed by the practices about preventing the transmission of infections and food poisoning. These findings were opposite with other studies conducted by Medeiros, et al., (2004) and Angolo, (2011) their studies revealed that, over-two thirds of the respondents correctly answered questions related to the following: practicing proper personal hygiene and preventing cross contamination. Participants' scores on specific questions related to cross contamination varied widely. The majority of the respondents correctly answered questions related to the practice most likely to results related to cross contamination.

According to these present study findings, before the educational program, the students had poor knowledge and practices about food safety; this was indicated by their low scores. This poor baseline scores which noticed among students' might be attributed to that, students abandon reading and neglect updating their professional knowledge. Another possible reason might be the absence of any resources or programs about food safety.

It was observed that, statistical significant differences were found between the mean score of knowledge and practices regarding the students' sociodemographic data. These findings were same as the findings of Roseman, and Kurzynske, (2006) who stated that, age, sex, income and educational levels influence food safety knowledge and behaviors of consumers.

Also findings reported by (Norazmir et al., (2012) showed that food safety knowledge is associated with the socio demographic and academic variable by 
increased food safety knowledge. while ByrdBredbenner et al., (2008) believed that there is not always a link between behaviors and knowledge and socio demographic. Also Angolo, (2011) stated that, no significant difference was found between food safety knowledge and sex of respondents between male and female respondents and no significant differences were found for self-reported food safety practices and demographic variables.

It could be observed that, male students who received the educational program had mean score of knowledge in post-test higher than the female students. This finding is in contract with Yasemin et al, (2013) who stated that female students who receive education have higher knowledge scores than their male counterparts. Also there were statistical significant difference between the post-test knowledge and students who prepare the food weekly, this finding in contract with Angolo, (2011) stated that, no significant $(\mathrm{p}<0.05)$ correlations were found between knowledge score and frequency of meal preparation.

The findings of the present study demonstrated, female students who received the educational program had mean score of practices in post-test higher than the male students. This finding same as results of Yasemin et al., (2013) who stated that female students who receive education have higher scores in their practices relating food safety compared to their male counterparts. Also females were found to have significantly higher practice pretest scores compared to males, and this finding is consistent with other research by Byrd-Bredbenner et al., (2008) and Bramlett (2011) who stated that, females have significantly higher practice pre-test scores compared to males .

Regarding to the mean score of knowledge and practice the present study revealed, no statistically significant differences between the study and control group in pre test but the food-safety knowledge and practices increased significantly between the study and control group in the post-test $(\mathrm{P}<0.001)$. After the program, the study showed statistically significant improvements for total knowledge and practice in relation to food safety. Thus, the improvement points to the effectiveness of the educational program, which was successful in students' acquisition of knowledge and improves their practice. The present study findings are in agreement with Angell (2008) who revealed that, food-safety knowledge increased significantly between pre and post test $(p<.0001)$. Also Medeiros et al., (2004) found that the mean "knowledge gain" scores for the intervention groups were significantly higher than for the control $(p<.01)$. In the current study, the majority of students $(80 \%)$ in the pre-test had got poor score of knowledge (less than $50 \%)$, (16\%) were satisfactory (between $50 \%$ $75 \%$ ), and only (4\%) had good knowledge (over $75 \%$ ), In post-test (after the educational program), $72 \%$ of the students had got good score of knowledge, $(27.5 \%)$ of them had satisfactory level and no students had poor knowledge. These findings in consistence with the findings of Angolo, (2011) who stated that, the change in knowledge score was found to be significant in all groups except the control group. Also the study of Angell (2008) revealed that food-safety knowledge increased significantly between pre and post test $(p<.0001)$. Also Gagger, et al., (2011) stated that, students' knowledge and awareness regarding food safety improved significantly after food safety education intervention.

Furthermore, from the findings it was observed that, in the study group, the majority of students (82\%) had got poor score of knowledge in the pre test and only $(3.5 \%)$ of them had good knowledge, while at the post- test $71.5 \%$ of them had got good score of knowledge and $(28.5 \%)$ of them had satisfactory level. No statistically significant differences between the pre test and post test practices in the control group before program but there were statistically significant differences found between the pre-test and post-test practices in the study group after program $\mathrm{P}=<0.001$. These findings in consistence with the findings of Angolo, (2011) who stated that, no significant differences were found among the study and control groups, practice, or knowledge scores at the time the pre-test was administered, Also these findings in consistence with the findings of Yarrow et al., (2009) who stated that, food safety beliefs, attitudes, knowledge and practices could change through educational intervention.

\section{Conclusions}

The study was useful in providing baseline assessment regarding the food safety knowledge, and self-reported practices of adolescent students, a group that is increasingly becoming important to reach because of their current and future roles as part of the entire Egyptian population.

The findings of this study concluded that, adolescent students have a problem with the knowledge and practices_related to food safety as personal hygiene, preventing cross contamination, keeping foods at safe temperatures and cooking foods adequately as illustrated in the base line assessment of their knowledge and practices about food safety.

The study has also shown the students' knowledge and practices about food safety were increased significantly among students who had the food safety 
educational program rather than the students who don't.

\section{Limitations of the study}

The study was limited by the use of convenience random sampling for specific age group. Since this type of sampling was used in the study, it may not be representative of the entire population. The results cannot be generalized to all students or to all ages.

\section{Recommendations}

The study recommended further research to explore students' beliefs, knowledge, and practices regarding food safety. In addition, further research about food safety educational programs should be taught among students that do not offer food safety program.

-Food safety should be addressed at an early stage in the educational institutions.

-Adequate books and magazines which include materials related to food safety should be available to the school.

-Establishment of teaching classes in each school to provide guideline for students about food safety

\section{References}

1- Abdel Tawab A., (2002): a measure of the level of social - economic status of families and key appreciation. Professor of Education and Dean of the Faculty of Education - Assiut University.

2- Ajello T., Doyle J., and Yeates M., (2014): Teacher Advisors Your Game Plan for Food Safety. The Partnership for Food Safety Education. www.foodsafety.gov.

3- Angell, D., (2008): Food Demonstration and Taste Testing Format: Ohio Extension Program Proves Effective", Journal of Family and Consumer Sciences, Vol. 100, p. 38.

4- Angolo, (2011): Food Safety Knowledge, Beliefs and Self-Reported Handling Practices of International College Students at a Midwestern University. Department of Hospitality Management and Dietetics. College of Human Ecology. Kansas State University.

5- Bramlett A., (2011): An Evaluation of the Use of Social Media for Food Safety Education. The University of Georgia.

6- Byrd-Bredbenner, C., Abbot, C., Wheatley, V., Schaffner, D., Bruhn, C., and Blalock, L., (2008): Risky Eating Behaviours of Young Adults - Implications for Food Safety Education. Journal of the American Dietetic Association, 108 , 549-552. http://dx.doi.org/10.1016/j.jada.2007.12.013.

7- Curtis, R., (2008): Food safety knowledge of undergraduate students at California state university, Fullertrton (Master's thesis).
Available from prorequest Dissertations and Theses database. (UMI No. 1462821).

8- Diane M., DeBacker, Dale M., Dennis, and Brad N., (2013): Food Safety for Volunteers \& Students. Kansas State Department of Education.

9- Gagger V., Atkinson C., and Jaroni D., (2011): Evaluating Food Safety Habits Among College Students. Human Nutrition, Health, Family and Consumer Sciences, Southern University Agricultural, Research and Extension Center, Ashford O. Williams Hall, Baton Rouge, LA 70813.

10- Haapala, I., \& Probart, C., (2004): Food safety knowledge, perceptions, and behaviors among middle school students. Journal of Nutrition Education and Behavior, 36, 71-76.

11- Jevsnik, M., Hlebec, V., and Raspor, P., (2008) Consumers' Awareness of Food Safety from Shopping to Eating. Food Control, 19, 737745.

http://dx.doi.org/10.1016/j.foodcont.2007.07.017

12- McArthur L., Holbert, and W., Forsythe. (2007): College students and awareness of food safety. J Fam. Cons. Sci. 99:60-67.

13- Medeiros, L., Hillers, V., Chen, G., Bergmann, V., Kendall, P. and Schroeder, M., (2004): "Design and development of food safety knowledge and attitude scales for consumer food safety education", Journal of the American Dietetic Association, Vol. 104, No. 11, pp. 1671-7.

14- Norazmir, M., Noor, H., Shafurah, S., Shafurah, S., Ajau, D., and Norazianshah, H., (2012): Knowledge and Practices on Food Safety among Secondary School Students in Johor Bahru, Johor Malaysia. Pakistan Journal of Nutrition, 11,110-115. http://dx.doi.org/10.3923/pjn.2012.110.115

15- Redmond, E., \& Griffith, C., (2005): Consumer perceptions of food safety risk, control and responsibility. Appetite, 43(3), 309313.

16- Roseman, M., and Kurzynske, J., (2006): Food Safety Perceptions and Behaviours of Kentucky Consumers. Journal of Food Protection, 69, 1412-1421.

17- Spedigo A., Richards J., Saxton A., Souza D., and Draughon F., (2009): Characterization of food safety knowledge, attitudes, and behaviors of adolescents in East Tennessee. Food protection Trends, vol.29.No10.Page 626-642.

18- The Partnership for Food Safety Education (2013): Educational Program. Fight BAC! ${ }^{\circledR}$ At www.fightbac.org.

19- WHO. (2007): http://www.who.int/whr/2007/en/index.html. 
20- World Health Organization (2010): Prevention of food borne disease: Five keys to safer food. Retrieved 2010-12-10.

21- Yarrow L., Reming V., and Higging M., (2009): Food safety education intervention positively influences college students' food safety attitudes, beliefs, knowledge, and selfreported practices. J. of Environ. Health 71: 30.

22- Yasemin A., Huseyin G., and Isil S., (2013):

University Students' Knowledge and Practices of Food Safety. Anthropologist, 16(3): 677-684. 